\title{
Visual deviant stimuli produce mismatch responses in the amplitude dynamics of neuronal oscillations
}

\section{Tugin, Sergei}

2016-11-15

Tugin , S , Hernandez-Pavon , J C , Ilmoniemi , R J \& Nikulin , V V 2016 , ' Visual deviant stimuli produce mismatch responses in the amplitude dynamics of neuronal oscillations ', Neurolmage , vol. 142 , pp. 645-655 . https://doi.org/10.1016/j.neuroimage.2016.07.024

http://hdl.handle.net/10138/229298

https://doi.org/10.1016/j.neuroimage.2016.07.024

publishedVersion

Downloaded from Helda, University of Helsinki institutional repository.

This is an electronic reprint of the original article.

This reprint may differ from the original in pagination and typographic detail.

Please cite the original version. 


\title{
Visual deviant stimuli produce mismatch responses in the amplitude dynamics of neuronal oscillations
}

\author{
Sergei Tugin ${ }^{\mathrm{a}, \mathrm{b}, *, 1}$, Julio C. Hernandez-Pavon ${ }^{\mathrm{a}, \mathrm{b}, 1}$, Risto J. Ilmoniemi ${ }^{\mathrm{a}, \mathrm{b}}$, Vadim V. Nikulin ${ }^{\mathrm{c}, \mathrm{d}}$ \\ a Department of Neuroscience and Biomedical Engineering (NBE), Aalto University School of Science, Espoo, Finland \\ ${ }^{\mathrm{b}}$ BioMag Laboratory, HUS Medical Imaging Center, University of Helsinki and Helsinki University Hospital, Helsinki, Finland \\ ${ }^{c}$ Center for Cognition and Decision Making, National Research University Higher School of Economics, Russian Federation \\ ${ }^{\mathrm{d}}$ Neurophysics Group, Department of Neurology, Campus Benjamin Franklin, Charité, University Medicine, Berlin, Germany
}

\section{A R T I C L E I N F O}

\section{Article history:}

Received 24 February 2016

Received in revised form 21 June 2016

Accepted 10 July 2016

Available online 16 July 2016

\section{Keywords:}

Electroencephalography (EEG)

Event-related desynchronization (ERD)

Event-related potential (ERP)

Event-related synchronization (ERS)

Mismatch negativity (MMN)

Oscillations

Peripheral vision

Visual mismatch oscillatory response (VMOR)

\begin{abstract}
A B S T R A C T
Objectives: Auditory and visual deviant stimuli evoke mismatch negativity (MMN) responses, which can be recorded with electroencephalography (EEG) and magnetoencephalography (MEG). However, little is known about the role of neuronal oscillations in encoding of rare stimuli. We aimed at verifying the existence of a mechanism for the detection of deviant visual stimuli on the basis of oscillatory responses, so-called visual mismatch oscillatory response (vMOR).

Methods: Peripheral visual stimuli in an oddball paradigm, standard vs. deviant (7:1), were presented to twenty healthy subjects. The oscillatory responses to an infrequent change in the direction of moving peripheral stimuli were recorded with a 60-channel EEG system. In order to enhance the detection of oscillatory responses, we used the common spatial pattern (CSP) algorithm, designed for the optimal extraction of changes in the amplitude of oscillations.

Results: Both standard and deviant visual stimuli produced Event-Related Desynchronization (ERD) and Synchronization (ERS) primarily in the occipito-parietal cortical areas. ERD and ERS had overlapping timecourses and peaked at about 500-730 ms. These oscillatory responses, however, were significantly stronger for the deviant than for the standard stimuli. A difference between the oscillatory responses to deviant and standard stimuli thus reflects the presence of vMOR.

Conclusions: The present study shows that the detection of visual deviant stimuli can be reflected in both synchronization and desynchronization of neuronal oscillations. This broadens our knowledge about the brain mechanisms encoding deviant sensory stimuli.
\end{abstract}

(c) 2016 Elsevier Inc. All rights reserved.

\section{Introduction}

Rare deviant stimuli are biologically important, as they usually indicate, in an otherwise predictable environment, a change that can lead to behaviorally relevant consequences (Sokolov et al., 2002). In the human brain, such changes in stimulus properties can be detected electrophysiologically via recording of

Abbreviations: CSP, common spatial pattern; EEG, electroencephalography; EOG, electrooculography; ERD, event-related desynchronization; ERP, event-related potential; ERS, event-related synchronization; MEG, magnetoencephalography; MMN, mismatch negativity; MOR, mismatch oscillatory response; ROI, region of interest; vMMN, visual mismatch negativity; vMOR, visual mismatch oscillatory response.

* Corresponding author at: Aalto University School of Science, Department of Neuroscience and Biomedical Engineering (NBE) P.O. Box 12200, FI-00076 AALTO, Finland.

E-mail address: sergei.tugin@aalto.fi (S. Tugin).

1 These authors have contributed equally to the work. evoked responses. The auditory mismatch negativity (MMN) is a classic example of deviant stimulus detection (for reviews, see Näätänen et al., 2011, 2007). Analogously, MMN to visual stimuli can also be recorded (visual mismatch negativity, vMMN); it is a negative event-related potential with a posterior/occipito-parietal scalp distribution peaking at around $150-400 \mathrm{~ms}$ after the onset of deviant visual stimuli (Kimura, 2012). The vMMN can be elicited with an oddball paradigm, where infrequent stimuli are semi-randomly embedded among frequent standard stimuli (Kimura, 2012; PazoAlvarez et al., 2003; Stefanics et al., 2012, 2014). vMMN is sensitive to deviant stimulus characteristics such as changes in contrast, color, shape, movement direction, form, orientation, or spatial frequency (Czigler, 2014; Kimura, 2012; Pazo-Alvarez et al., 2003; Stefanics et al., 2012).

Although traditional evoked mismatch responses have been found in auditory and visual modalities, it is less clear whether deviant stimuli can in addition produce changes in the amplitude 
dynamics of auditory (Fuentemilla et al., 2008; Hsiao et al., 2009; Ko et al., 2012) or visual neuronal oscillations (Stothart and Kazanina, 2013). As evoked responses and neuronal oscillations represent two major modes of brain functioning (Roach and Mathalon, 2008), answering this question is important for understanding neuronal processing responsible for change detection.

It would be particularly important to show whether alpha oscillations can produce mismatch responses to deviant stimuli. This is because the alpha rhythm has been shown to reflect inhibitory processes in the cortex; thus, a change in its amplitude would relate to patterns of excitation/inhibition caused by the deviant stimuli. An increase in the amplitude of oscillations (also referred to as eventrelated synchronization, ERS (Pfurtscheller and Lopes da Silva, 1999)) indicates the presence of task-related inhibitory activity in a given area (Jensen et al., 2014; Jensen and Mazaheri, 2010; Palva and Palva, 2007), while an attenuation of oscillatory amplitude (also referred to as event-related desynchronization, ERD) indicates an underlying active process, characterized by increased excitation (Pfurtscheller and Lopes da Silva, 1999; Sauseng et al., 2005; Thut et al., 2012).

In the present study, we addressed the question of whether the changes in the strength of alpha-rhythm ERD/ERS can reflect a neuronal response to deviant stimuli and whether in fact both desynchronization and synchronization can occur simultaneously. Given that we studied responses to visual stimuli, we refer below to the changes in the oscillations to deviant stimuli as visual mismatch oscillatory responses (vMOR).

An important factor to be considered is the multitude of oscillatory processes in the alpha frequency range, which are heavily superposed on the sensor level in EEG or MEG (Haufe et al., 2014). In order to enhance the detection of oscillatory responses to deviant stimuli, we used an optimization algorithm (common spatial patterns, CSP; Blankertz et al., 2008) for the efficient extraction of oscillatory components.

\section{Method}

\section{Subjects}

Twenty healthy volunteers (11 females, 9 males; $28 \pm 3$ years; 1 left-handed and 19 right-handed) with normal or corrected-tonormal vision were enrolled. None of the subjects had neurological diseases. The experimental protocol was approved by the Ethics Committee of Helsinki University Hospital and was in compliance with the Declaration of Helsinki. The subjects gave their written informed consent prior to the experiments.

\section{Stimuli}

Peripheral vision is important since it allows detection of unexpected or deviant signals and in many cases our survival depends on the ability to perceive the movement of significant objects (e.g., detecting peripheral driving vehicles on the road, avoiding a predator or a falling tree, etc.). Therefore, it is important to describe the neuronal mechanisms of such stimulation. Consequently, in this study, we use two displays to simulate a more realistic situation.

The subjects sat in a comfortable chair and were instructed to remain awake, to keep their eyes open and to focus on a white fixation cross presented on a black background in front of them. They were also advised to minimize any unnecessary movements and blinking. Two 21-inch full-HD screens were placed laterally at 80 $\mathrm{cm}$ from the medial head line. The viewing angles of each monitor ranged from $59^{\circ}$ (distal side) to $90^{\circ}$ (proximal side) (Fig. 1A).

White circular spots (radius of 150 pixels, $37 \mathrm{~mm}$, visual angle $2.65^{\circ}$ ) were presented simultaneously on both screens (one spot per screen). The spots moved in a circle with angular velocity of about $42 \mathrm{rpm}$, the direction of this rotary motion defining the

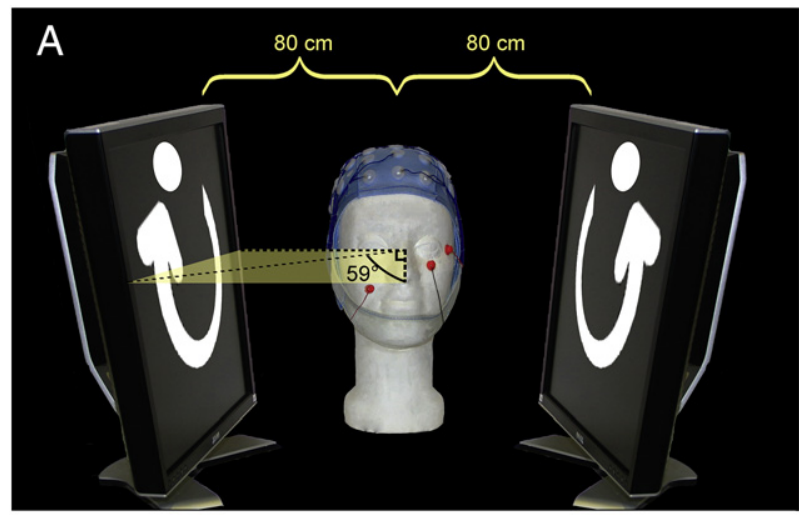

B

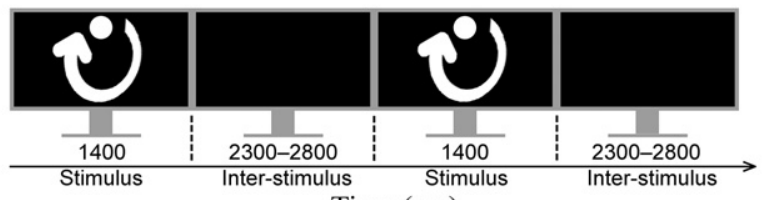

Time (ms)

Fig. 1. Experimental setup and stimulus paradigm. A) Two screens were placed laterally at $80 \mathrm{~cm}$ from the medial head line. B) The stimulus duration was $1400 \mathrm{~ms}$; the inter-stimulus interval varied randomly between 2300 and $2800 \mathrm{~ms}$.

difference between the standard and deviant stimuli. The direction of the motion was randomized among subjects. From 657 to $837(800 \pm 8)$ stimuli were presented semi-randomly in an oddball paradigm with a $1: 7$ ratio, i.e., 81 to $100(98 \pm 1)$ deviants and 576 to $737(702 \pm 8)$ standards. The stimulus duration was 1400 ms which corresponds to one rotation of the spots. The interstimulus interval was randomized between 2300 and $2800 \mathrm{~ms}$ (Fig. 1B).

\section{EEG recordings}

The electric potentials on the scalp were recorded with a 60channel eXimia EEG (Nexstim Plc., Helsinki, Finland), electrodes being placed according to the international 10-20 system. The signals were referenced to the right mastoid; the ground electrode was over the right cheek bone. Eye movements were recorded with two additional electrodes; one electrooculography (EOG) electrode was placed on the frontal process of the zygomatic bone and the other under the eye (Fig. 1A). The impedance of all electrodes was kept below $5 \mathrm{k} \Omega$. The signals were band-pass filtered from 0.1 to $350 \mathrm{~Hz}$ and digitized at $1450 \mathrm{~Hz}$. The EEG was recorded for about $90 \mathrm{~min}$ with a 1-minute break after every 5 min of recording.

\section{Electrooculography}

The individual EOGs were processed with band-pass (0.5$40 \mathrm{~Hz}$ ) and notch filters $(48-52 \mathrm{~Hz})$ to remove high-frequency and the remaining power-line interference, respectively. Both filters were fourth-order, Butterworth, and zero-phase. The data of eighteen individual EOGs were averaged for standard and deviant stimuli; data of two subjects were excluded due to poor EOG quality (i.e., excessive noise and artifacts).

\section{Data analysis}

Offline analysis was performed with Matlab (The Mathworks, Inc., Natick, Massachusetts, USA). The EEG recordings were visually inspected. The epochs with amplitudes larger than $\pm 100 \mu \mathrm{V}$ or 
containing excessive muscle activity (i.e., from scalp, neck muscle or blinks) were removed from the analysis. On average, $514 \pm 6$ epochs were included in the analysis; $65 \pm 1$ deviants and $449 \pm 6$ standards. The bad channels (e.g., those with strong muscle/eye movements or with poor electrode contact) were excluded from the analysis; a channel was rejected if it had an amplitude larger than $\pm 100 \mu \mathrm{V}$ or presented excessive muscle activity or was disconnected. After removing bad epochs and bad channels, the EEG measurement data matrix $\mathbf{Y} \in \mathbb{R}^{m \times N}$ consisted of $m$ channels and $N$ time points, $N=n \times T$, where $n=n_{s}+n_{d}$ is the remaining number of epochs ( $n_{s}$ remaining standard plus $n_{d}$ remaining deviant) and $T$ the number of time points per epoch. The remaining data matrix was filtered in alpha-band frequency $(8-12 \mathrm{~Hz})$ using a fourth-order, Butterworth, zero-phase band-pass filter; the filtered data were centered to the mean of all remaining data, resulting in the data matrix $\mathbf{X} \in \mathbb{R}^{m \times N}$.

$\mathbf{X}$ was divided in two data matrices, $\mathbf{X}_{\mathrm{s}}$ containing standard epochs ( -1000 to $3000 \mathrm{~ms})$ and $\mathbf{X}_{\mathrm{d}}$ containing deviant ones $(-1000$ to $3000 \mathrm{~ms}$ ). To avoid effects of overfitting, the number of standard epochs contained in $\mathbf{X}_{\mathrm{s}}$ was equalized to the number of deviant epochs in $\mathbf{X}_{\mathrm{d}}$, the standard epochs were uniformly pseudo-randomly distributed with the function "randperm" in Matlab. Thereafter, each epoch in $\mathbf{X}_{\mathrm{s}}$ and $\mathbf{X}_{\mathrm{d}}$ was divided into pre-stimulus ( -1000 to $0 \mathrm{~ms}$ ) and post-stimulus (100 to $1000 \mathrm{~ms}$ ) segments, see Fig. 2. We excluded the first $100 \mathrm{~ms}$ since there is evidence that early latencies are not involved in the generation of vMMN and only latencies larger than $100 \mathrm{~ms}$ are involved in high-level processing of the stimuli such as identification of movement direction (Amano et al., 2006; Lamberty et al., 2008). The pre-stimulus segments corresponding to the standard data were concatenated, for each subject separately, in a matrix $\mathbf{X}_{\text {spre }}$; similarly, the pre-stimulus segments for the deviant data were concatenated in $\mathbf{X}_{\text {dpre }}$. A similar procedure was applied for the post-stimulus segments for both standard and deviant conditions, resulting in matrices $\mathbf{X}_{\mathrm{spos}}$ and $\mathbf{X}_{\mathrm{dpos}}$, respectively.

\section{Common spatial patterns}

The common spatial pattern (CSP) algorithm is an effective supervised data-driven method to study multichannel data recorded in different conditions (Blankertz et al., 2008). CSP can decompose multivariate signals recorded from two classes or conditions so that the decomposed sources have maximal discriminability (in the sense of variance ratio) between the conditions. This implies that CSP finds spatial filters that maximize the difference in power between the conditions. CSP can be applied for a wide range of stimulus conditions, for instance contrasting direction, frequency, intensity of the stimuli or contrasting pre- and post-stimulus intervals.

In CSP, the variance in one condition is maximized while the variance in the other condition is minimized at the same time using spatial filters. The solution of the CSP is determined by the simultaneous diagonalization of the two covariance matrices, which is equivalent to solving the generalized eigenvalue problem. Therefore, CSP is optimal for the extraction of changes in the amplitude of the oscillations from pre- and post-stimulus responses. CSP projects the signal matrix $\mathbf{X}$ through a matrix of spatial filters $\mathbf{W}$ leading to a new signal matrix $\mathbf{X}_{\mathrm{CSP}}=\mathbf{W}^{\mathrm{T}} \mathbf{X}$.

In the following, we describe the steps carried out to compute CSP. First, covariance matrices are computed for pre- and poststimulus standard and deviant data:

$\mathbf{C}_{c}=\frac{1}{N_{c}} \mathbf{X}_{c} \mathbf{X}_{c}^{\mathrm{T}}$

where $\mathbf{C}_{c}$ is the covariance matrix corresponding to condition $c \in$ \{spre, spos, dpre, dpos\}, and $N_{c}$ is the number of time points in condition $c$. Thereafter, the generalized eigenvalue problem is solved to compute the spatial filters and patterns that maximize the power difference between pre- and post-stimulus intervals for both conditions.

$$
\begin{aligned}
& \mathbf{C}_{\mathrm{spre}} \mathbf{W}_{\mathrm{s}}=\boldsymbol{\lambda}_{\mathrm{s}} \mathbf{C}_{\mathrm{spos}} \mathbf{W}_{\mathrm{s}}, \\
& \mathbf{C}_{\mathrm{dpre}} \mathbf{W}_{\mathrm{d}}=\boldsymbol{\lambda}_{\mathrm{d}} \mathbf{C}_{\mathrm{dpos}} \mathbf{W}_{\mathrm{d}},
\end{aligned}
$$

where $\mathbf{W}_{\mathrm{s}}$ and $\mathbf{W}_{\mathrm{d}}$ are $m \times m$ matrices of filters or de-mixing matrices for the standard and deviant data, respectively. Furthermore, $\boldsymbol{\lambda}_{\mathrm{S}}$ and $\lambda_{\mathrm{d}}$ are the matrices of eigenvalues for standard and deviant data, respectively. The mixing matrices or matrices of spatial patterns for standard $\mathbf{A}_{\mathrm{s}}$ and deviant $\mathbf{A}_{\mathrm{d}}$ data are computed as follows;

$$
\begin{aligned}
& \mathbf{A}_{\mathrm{s}}=\left(\mathbf{W}_{\mathrm{s}}^{-1}\right)^{\mathrm{T}}, \\
& \mathbf{A}_{\mathrm{d}}=\left(\mathbf{W}_{\mathrm{d}}^{-1}\right)^{\mathrm{T}} .
\end{aligned}
$$

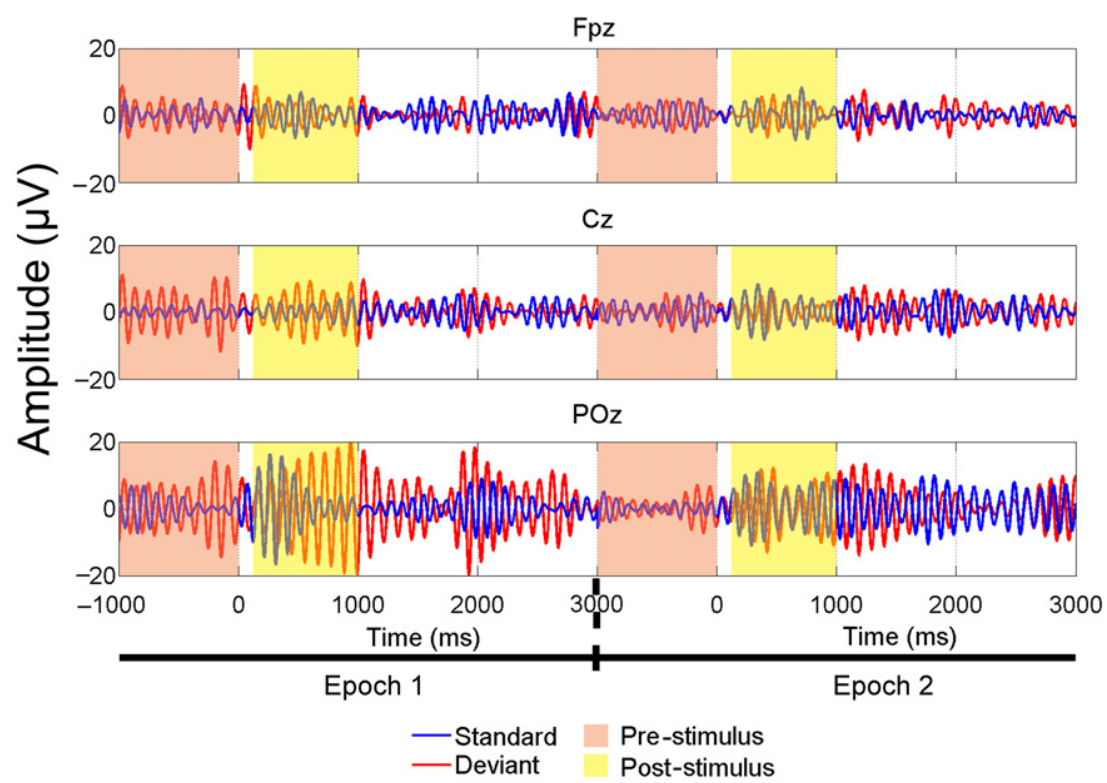

Fig. 2. Example of two epochs for standard and deviant stimuli after filtering (Subject 1). The pre- and post-stimulus intervals are shown. 
Then, the common spatial components are computed by projecting the centered data matrix $\mathbf{X}$ using spatial filters $\mathbf{W}_{\mathrm{s}}$ and $\mathbf{W}_{\mathrm{d}}$ :

$\mathbf{P}_{\mathrm{s}}=\mathbf{W}_{\mathrm{s}}^{\mathrm{T}} \mathbf{X}$

$\mathbf{P}_{\mathrm{d}}=\mathbf{W}_{\mathrm{d}}^{\mathrm{T}} \mathbf{X}$

where $\mathbf{P}_{\mathrm{s}}$ and $\mathbf{P}_{\mathrm{d}}$ are $m \times N$ matrices of common spatial components for standard and deviant data, respectively.

\section{Event-related desynchronization/synchronization}

Event-related desynchronization (ERD) and event-related synchronization (ERS) describe the amplitude modulation of ongoing activity induced by external stimuli or events (Pfurtscheller and Lopes da Silva, 1999). ERD and ERS refer to the decrease and increase of the instantaneous oscillatory amplitude, respectively. ERD and ERS show different dynamics and sensitivity depending on the frequency band in which they are obtained.

The instantaneous amplitude was obtained by computing the absolute values of the analytic signal corresponding to the common spatial components obtained from Eq. (4),

$\mathbf{E}_{\mathrm{s}}=\left|\mathbf{P}_{\mathrm{s}}(j)+i H\left(\mathbf{P}_{\mathrm{s}}(j)\right)\right|$,

$\mathbf{E}_{\mathrm{d}}=\left|\mathbf{P}_{\mathrm{d}}(j)+i H\left(\mathbf{P}_{\mathrm{d}}(j)\right)\right|$,

where $\mathbf{P}_{\mathrm{s}}(j)=\mathbf{P}_{\mathrm{s}}(j,:)$ and $\mathbf{P}_{\mathrm{d}}(j)=\mathbf{P}_{\mathrm{d}}(j,:), j=1,2, \ldots, m, \mathbf{P}_{\mathrm{s}}(j,:)$ and $\mathbf{P}_{\mathrm{d}}(j,:)$ denote the $j$ : th row of $\mathbf{P}_{\mathrm{s}}$ and $\mathbf{P}_{\mathrm{d}}$, respectively. Furthermore, $\mathbf{E}_{\mathrm{s}}$ and $\mathbf{E}_{\mathrm{d}}$ are $m \times N$ matrices of event-related responses, which represent amplitude envelopes of the signals $\mathbf{P}_{\mathrm{s}}(j)$ and $\mathbf{P}_{\mathrm{d}}(j)$, respectively, and $H$ is the Hilbert transform.

Thereafter, $\mathbf{E}_{\mathrm{s}}$ and $\mathbf{E}_{\mathrm{d}}$ were epoched, yielding $\mathbf{E}_{\mathrm{s}}^{\prime}$ and $\mathbf{E}_{\mathrm{d}}^{\prime}$ for standard and deviant epochs, respectively. The averages over epochs, $\mathbf{R}_{\boldsymbol{s}}$ and $\mathbf{R}_{\mathrm{d}}$, were obtained for $\mathbf{E}_{\mathrm{s}}^{\prime}$ and $\mathbf{E}_{\mathrm{d}}^{\prime}$, respectively. The normalized event-related responses were computed as follows:

$\mathbf{E}_{\mathrm{s}}(j, t)(\%)=\frac{\mathbf{R}_{\mathrm{s}}(j, t)-\mathbf{R}_{\mathrm{sRef}}(j)}{\mathbf{R}_{\mathrm{sRef}}(j)} \times 100$,
$\mathbf{E}_{\mathrm{d}}(j, t)(\%)=\frac{\mathbf{R}_{\mathrm{d}}(j, t)-\mathbf{R}_{\mathrm{dRef}}(j)}{\mathbf{R}_{\mathrm{dRef}}(j)} \times 100$,

where $j=1,2, \ldots, m$ and $t=0$ to 3000 ms. $\mathbf{R}_{\text {sRef }}(j)$ and $\mathbf{R}_{\mathrm{dRef}}(j)$ are the means of $\mathbf{R}_{\mathrm{S}}$ and $\mathbf{R}_{\mathrm{d}}$ over the time interval -1000 to $0 \mathrm{~ms}$, respectively.

The first components of the event-related responses, obtained from Eq. (6), contain the minimum variance in the post-stimulus interval indicating ERD, whereas the last components indicate ERS and contain the maximum variance.

Note that the CSP algorithm was applied separately for the data corresponding to the standard and deviant stimuli. In this way, differences in ERD/ERS between the conditions (standard vs. deviant stimuli) could not have been due to overfitting, since the CSP algorithm was not applied to both classes simultaneously. For each subject, CSP analysis was repeated 100 times by iteratively equalizing the number of standard epochs to the number of deviant epochs. This way we used different standard epochs in each iteration while keeping their number identical to the number of deviant epochs. The results in each of these iterations were then averaged to obtain final ERD and ERS components. The rationality behind this was to use all epochs in the study and avoid random effects by selecting just a few of them. By iteratively selecting the epochs 100 times we increased the robustness of the outcome. We also calcu- lated differences between ERD and ERS curves belonging to deviant and standard stimuli within each of the above-mentioned iterations. We compared these results with the results where the standard and deviant categories were permuted in different subjects. Using Fisher's exact test, we then calculated the probability of obtaining significant $p$-values $(p<0.05)$ when comparing ERD/ERS responses belonging to standard and deviant stimuli versus a comparison of ERD/ERS responses belonging to the permuted data.

\section{Sensor space}

The ERD/ERS values were calculated for each electrode separately. For this analysis, after removing bad channels and trials from the measurement data, the signal from each electrode was re-referenced to the average of all channels (common average electrode). Thereafter, the data were filtered and centered as was described in Data analysis section. For calculating the oscillatory responses, the instantaneous amplitude was obtained by computing the absolute values of the analytic signal corresponding to the data $\mathbf{X}$, similarly to that in Eq. (5). The ERD and ERS were studied for standard and deviant stimuli by using Eq. (6). No CSP was applied for the sensor space analysis.

\section{Analysis of event-related potentials}

For the event-related potential (ERP) analysis, the data were rereferenced to the common average electrode and the baseline was corrected in the pre-stimulus interval $(-100$ to $0 \mathrm{~ms})$. The data were band-pass-filtered in the $0.5-40 \mathrm{~Hz}$ frequency range; in addition, a notch filter (48-52 Hz) was applied to remove the remaining powerline interference. Both filters were fourth-order, Butterworth, and zero-phase. The data were averaged separately for the standard and deviant stimuli. The average response to standard stimuli was subtracted from that to the deviant ones. Ten channels were used for the analysis: $01, \mathrm{Oz}, \mathrm{O} 2, \mathrm{P} 3, \mathrm{P} 4, \mathrm{P} 5, \mathrm{P} 6, \mathrm{PO} 3, \mathrm{POz}$, and PO4. The analysis was performed for 1 ) individual channels and 2) for regions of interest (ROIs) where we averaged activity from the selected channels. Occipital (01, Oz, and O2) and parietal (P3, P4, P5, P6, PO3, POz, and PO4) ROIs were considered. The analysis and selection of the channels were performed according to the previous vMMN studies (Pazo-Alvarez et al., 2004; Kuldkepp et al., 2013).

\section{Behavioural assessment of attention}

The subjects were instructed to ignore the stimuli. To assess whether or not the subjects paid attention to the stimuli, we devised a procedure where subjects were asked three questions. This procedure was performed only in nine subjects. The questions were: 1) What kind of stimuli did you observe? 2) Which direction of the stimuli was most frequent? 3) What was the ratio of the stimuli? The EEG data from these subjects were analyzed in the same manner as for the previously recorded subjects. As a control condition, the same subjects were asked these questions but this time they were instructed to pay attention to the stimuli during 5 min and no EEG was measured. The control answers were compared to those when the subjects were instructed to ignore the stimuli.

\section{Statistical analysis}

The statistical analysis of ERD/ERS was performed for peak amplitudes and mean amplitudes (areas). The ERD/ERS peak amplitudes were determined separately for each subject in the time interval 300-900 ms, independently for standard and deviant responses. Note that we have not selected peaks in the time intervals where the grand-average had the largest differences. Instead, our time interval was selected to be broad enough to contain the 
ERD/ERS responses to standard and deviant stimuli. This selection of the time interval is based on previous studies showing that the first $1000 \mathrm{~ms}$ are most relevant for ERD/ERS during the presentation of visual stimuli (Babiloni et al., 2004; Bočková et al., 2013). Our subsequent latency analysis has demonstrated that the peaks for ERD/ERS were in the 300-900-ms time range. Furthermore, the peak latencies of the min/max values for ERD/ERS, respectively, were calculated as well. For the ERP analysis, the mean amplitudes of the resulting waveforms were calculated in the parietal and occipital areas for each single channel and for each ROI. For both individual channels and ROIs containing ERPs, the statistical comparisons were performed in the time interval $100-400 \mathrm{~ms}$ in 20 -ms windows translated in $20-\mathrm{ms}$ steps (Kuldkepp et al., 2013; Pazo-Alvarez et al., 2004). The peak amplitudes, mean amplitudes, and peak latencies for ERD/ERS and ERP were analyzed with the non-parametric Wilcoxon signed-rank test for paired observations, because some variables were not normally distributed according to the Lilliefors test. The significance level was set at $5 \%(p<0.05)$.

\section{Results}

\section{Visual mismatch oscillatory responses}

We observed amplitude dynamics traditionally associated with ERD (amplitude decrease) and ERS (amplitude increase) induced by the peripheral visual stimuli; statistical analysis was performed across subjects. Fig. 3 shows an example of ERD/ERS time-courses and corresponding topographies for a representative subject for deviant and the average of 100 iterations for standard stimuli. In all subjects, the strongest decrease (ERD) and increase (ERS) of alpha oscillations peaked on average at 500 and $730 \mathrm{~ms}$, respectively. The most important aspect of Fig. 3 is stronger ERD/ERS for the deviant than standard stimuli. The topographies corresponding to the strongest ERD/ERS components, for both standard and deviant stimuli, showed an occipito-parietal distribution. While Fig. 3 shows results for only one subject, the general tendency for ERD and ERS values being stronger for deviant than standard stimuli was further confirmed with statistical analysis. Figs. 4A and 4C show the mean of ERD/ERS components for all subjects for deviant and the average of 100 iterations for standard stimuli. The difference between the components belonging to two stimuli is shaded, this difference thus being a manifestation of vMOR. Since the topographies of CSP components have subject-specific distributions, for averaged topographies we used their absolute values. While this approach eliminated polarity, it made it possible to see the maxima of the spatial distributions across subjects. Figs. 4B and 4D show that for the strongest ERD and ERS components, respectively, these maxima were primarily located over the occipito-parietal areas, with some additional contribution from the central areas.

Fig. 5 shows the results of the statistical analysis for the differences in ERD/ERS between the two stimuli when the activities were averaged across the strongest components for all subjects and the average of 100 iterations for standard stimuli. On average, ERD peak amplitude was larger for deviant (48\%) than standard stimuli (40\%), this difference being significant $(p<0.001)$. The ERS magnitude was also significantly larger for deviant than standard stimuli, being $92 \%$ and $70 \%(p<0.001)$, respectively. The mean amplitudes were also statistically larger to deviant than to standard stimuli for both ERD/ERS, $(p=0.010)$ and ( $p=0.012)$, respectively. This analysis confirmed stronger decrease and increase of alpha oscillations to deviant compared to standard stimuli.

In addition, Fisher's exact test, related to individual iterations (see Event-related desynchronization/synchronization section), showed that the significant differences in ERD/ERS between two categories of stimuli occurred more often than for the permuted data $\left(p<10^{-25}\right)$ and in case of the not permuted data, responses to deviant stimuli were stronger.

Fig. 6 shows the peak latencies of oscillatory responses where the strongest ERD and ERS components were analyzed across subjects and for the average of 100 iterations for standard stimuli. The maximum of the ERD to standard and deviant stimuli was located at 534 and $664 \mathrm{~ms}$, respectively ( $p=0.003$ ). The latencies for ERS were also significantly shorter for standard (599 ms) than for deviant (694 ms) stimuli $(p=0.044)$.

Fig. 6 shows that compared to the responses to deviant stimuli, standard stimuli induced earlier ERD/ERS responses. In addition, the ERD induced by either of the stimuli was earlier than the corresponding ERS.

\section{Analysis in sensor space}

We also compared results obtained with CSP to ERD/ERS values calculated for each electrode separately. Fig. 7A shows the corresponding time-courses of oscillatory responses in all electrodes across subjects. Fig. 7B depicts the topographies of the mean amplitudes of ERD/ERS in the interval 300-900 ms.

The peak amplitudes across channels of ERD and ERS were stronger for deviant than standard stimuli, $p=0.017$ and $p<$ 0.001 , respectively. However, a statistical analysis did not reveal any significant differences in the mean amplitude (area) of ERD/ERS between standard and deviant stimuli. The peak latencies of ERS to standard stimuli were significantly shorter than the ERS latency to the deviant stimuli ( $p=0.016$ ), but there were no statistically significant differences between the peak latencies of ERD standard and deviant stimuli.
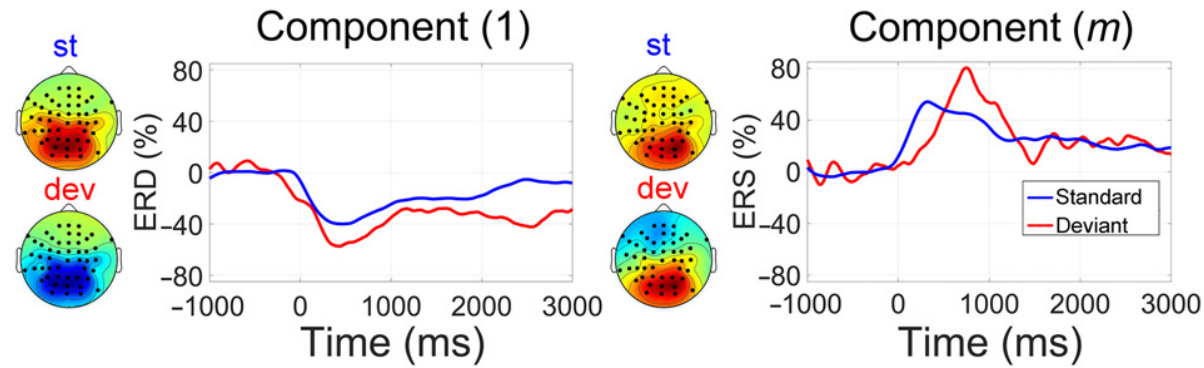

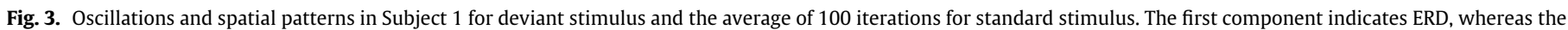
last component represents ERS. All topographies are on the same scale, $($ st $=$ standard, dev $=$ deviant). 


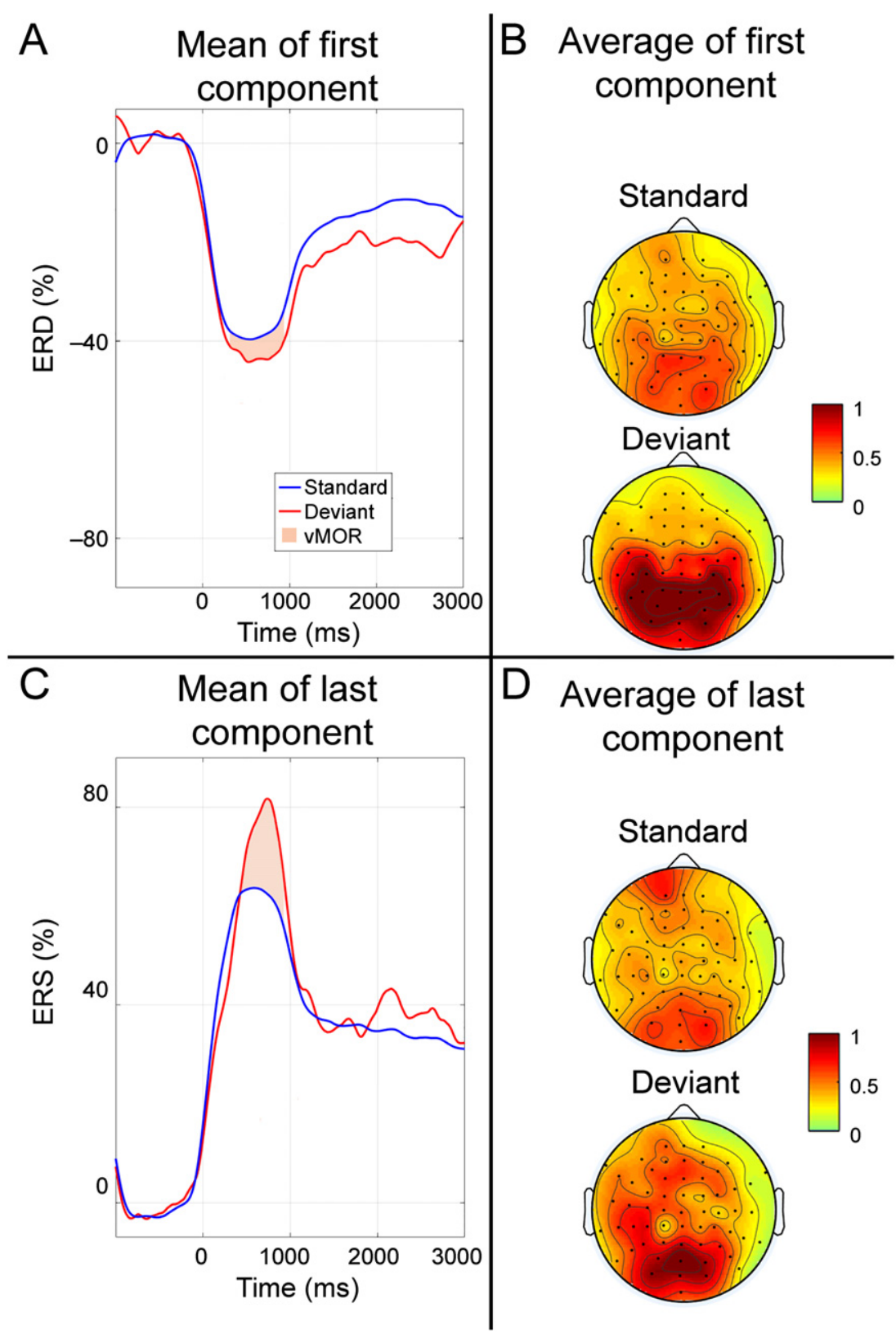

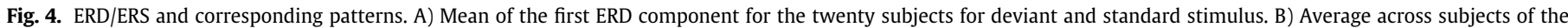

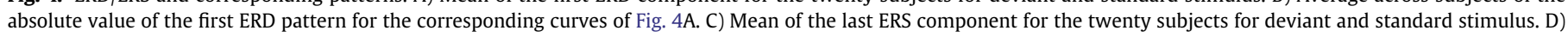
Average across subjects of the absolute value of the last ERS pattern for the corresponding curves of Fig. 4C. All topographies are on the same scale.

\section{Analysis of EOG}

No statistically significant differences in the mean amplitudes (area) between the ERPs to standard and deviant stimuli at EOG channels were found in the relevant time intervals: the beginning of stimulus presentation (0-110 ms), interval where vMOR was calculated (300-900 ms) and interval of the most prominent vMOR peaks (500-730 ms).

\section{Analysis of event-related potential}

Fig. 8A shows a grand-average ERP for all channels across subjects in the time interval from -100 to $600 \mathrm{~ms}$. Figs. $8 \mathrm{~B}-\mathrm{C}$ depict the
ROIs for occipital and parietal areas. A statistical analysis did not reveal any significant differences in the mean amplitude of any of the analyzed 20 -ms time intervals to standard and deviant stimuli $(p>0.05)$ in individual channels and in the corresponding ROIs.

\section{Behavioural assessment of attention}

Most of the subjects were unable to give a good estimate of the correct ratio of deviant and standard stimuli when being instructed to pay no attention to the stimuli. Moreover, some subjects were not even able to identify a correct direction of the moving stimuli when they did not pay attention to the stimuli, see Table 1 . The perceived ratios varied from 1:1 to $1: 10$. Nevertheless, when the subjects were 


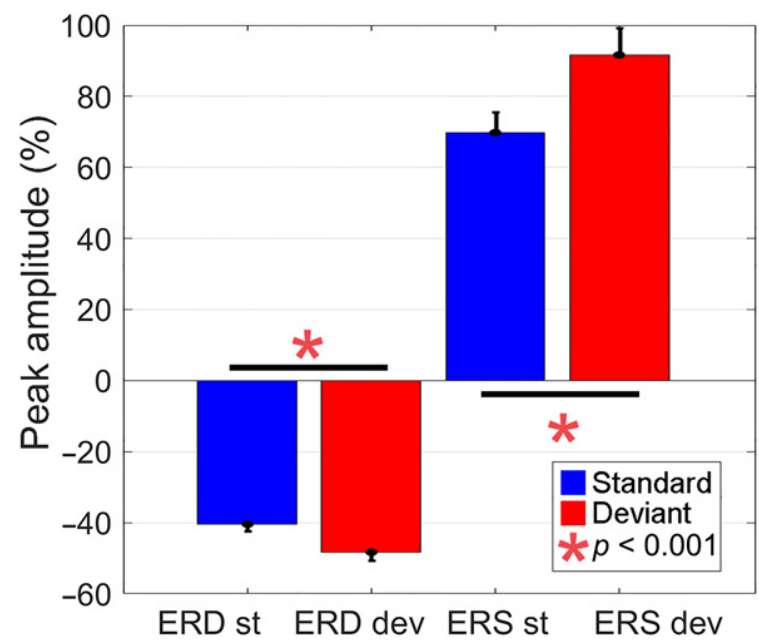

Fig. 5. Average of the peak amplitudes across the largest component of ERD/ERS to standard and deviant stimuli for all subjects. The error bars indicate the standard errors of the mean. There were statistically significant differences between the peak amplitudes of ERD/ERS for standard and deviant stimuli.

instructed to pay attention to the stimuli, the estimated ratios were very close to the actual ratios in the oddball sequence $1: 7$, Table 1. Neither the peak amplitude of ERD nor ERS showed dependency on the correct identification of the ratio (Fig. 9), which was further corroborated by the lack of the significant Spearman correlation between the peak amplitude of ERD/ERS and the perceived ratio. As another proof for the lack of the pronounced attentional effect on the strength of vMOR, we refer to the Supplementary Fig. 1 where the averaged oscillatory responses are shown for three subjects (S13, 15 and 16). Supplementary Fig. 1 shows that stronger ERD/ERS responses were recorded for deviant than standard stimuli despite the fact that these subjects were unable to detect at all the difference in the number of deviant and standard stimuli (1:1 reported ratio).

\section{Discussion}

In the present study, we focused on the detection of vMOR in alpha oscillations, which represent the most prominent oscillatory cortical phenomenon in the healthy awake human brain. Moreover, alpha oscillations have a high signal-to-noise ratio, which is important for the detection of even small changes in the amplitude dynamics. We have shown that vMOR is manifested in both decrease (ERD) and increase (ERS) of alpha oscillations to deviant compared to standard stimuli.

\section{Data-processing considerations}

Analysis in the sensor space is widely used to study evoked responses and oscillations. However, the spatial specificity of such

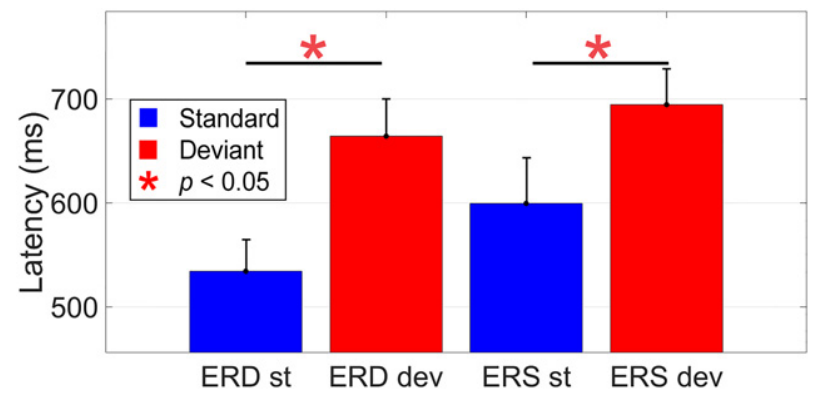

Fig. 6. Peak latencies across the largest component of ERD/ERS to standard (st) and deviant (dev) stimuli for all subjects. analysis is quite limited since multiple oscillatory processes are usually heavily superposed on the sensor level in EEG or MEG. Thus, sources of interest might be masked by some irrelevant stronger sources. To overcome multiple sources overlapping in the sensor space, the CSP algorithm was used in order to enhance the detection of oscillatory responses.

Only the first and last CSP components were analyzed. This was done in order to obtain the strongest oscillatory responses for ERD and ERS. The obtained results showed that deviant stimuli resulted in stronger ERD and ERS responses compared to the standard stimuli. Moreover, the differences in ERD/ERS between the conditions (standard vs. deviant stimuli) are unlikely to be due to overfitting, since the CSP algorithm was not applied to both classes simultaneously in order to maximize the differences between the oscillatory responses to standard and deviant stimuli. Instead, the CSP algorithm was applied for pre- vs. post-stimulus data separately for the data corresponding to the standard and deviant stimuli.

\section{Mismatch detection in neuronal oscillations}

Only a few EEG/MEG studies have been published on auditory MOR (mismatch oscillatory response, (Fuentemilla et al., 2008; Hsiao et al., 2009; Ko et al., 2012); these authors referred to their responses as auditory oscillatory MMN). In those articles, non-phase-locked and phase-locked responses were studied using event-related spectral perturbation (ERSP) and inter-trial coherence (ITC), respectively. Here we discuss the results relating to the induced activity (ERSP analysis is equivalent to ERD/ERS). It was shown that auditory MOR in frontal regions has an increase in theta power, without changes in temporal sites (Fuentemilla et al., 2008); furthermore, an increase in theta power has also been found in both frontal and central areas (Ko et al., 2012). In contrast, the results, obtained with MEG, have revealed an increase of theta power in temporal but not in frontal regions (Hsiao et al., 2009). All these studies have shown stronger ERS responses for deviant compared to standard stimuli in theta frequency band, suggesting that theta activity plays an important role in the generation of auditory MOR. Deviant stimuli seem to induce significant changes in power increase of theta and alpha broad-band (Fuentemilla et al., 2008; Ko et al., 2012). However, no statistically significant changes in the amplitude dynamics of alpha oscillations between standard and deviant stimuli have been observed in the three above-mentioned studies. This might relate to the fact that alpha oscillations are not particularly pronounced in the auditory cortical areas and thus any detection of oscillatory changes, induced by deviant stimuli, might be problematic due to low signal-to-noise ratio. Thus, only theta oscillatory activity was found to relate to the auditory sensory memory.

Similarly, it has recently been investigated whether induced theta activity plays a similar role in the generation of oscillatory visual MMN as it does in oscillatory auditory MMN (Stothart and Kazanina, 2013). The results by Stothart and Kazanina (2013) showed the reduction of alpha power to both standard and deviant stimuli in occipito-parietal sites. Importantly, the decrease of alpha power was stronger to deviant stimuli like in our study. However, in contrast to Stothart and Kazanina, we also found a stronger increase of alpha oscillations (ERS) to deviant than standard stimuli. In our study, the activation of ERD/ERS showed a broad spatial distribution; however, the responses rather had topographies with a clear maximum over occipito-parietal regions, which is in line with ERP vMMN studies (Kimura, 2012) and the study of Stothart and Kazanina (2013).

In contrast to other studies where changes in motion and different types of peripheral stimulation have been used (Amenedo et al., 2007; Kuldkepp et al., 2013), in our study, peripheral stimulation ( $59^{\circ}$ with respect to the fixation cross) induced only weak responses, see Fig. 8. Probably due to the small ERP, our analysis did not reveal statistically significant differences between evoked responses to the 


\section{A}
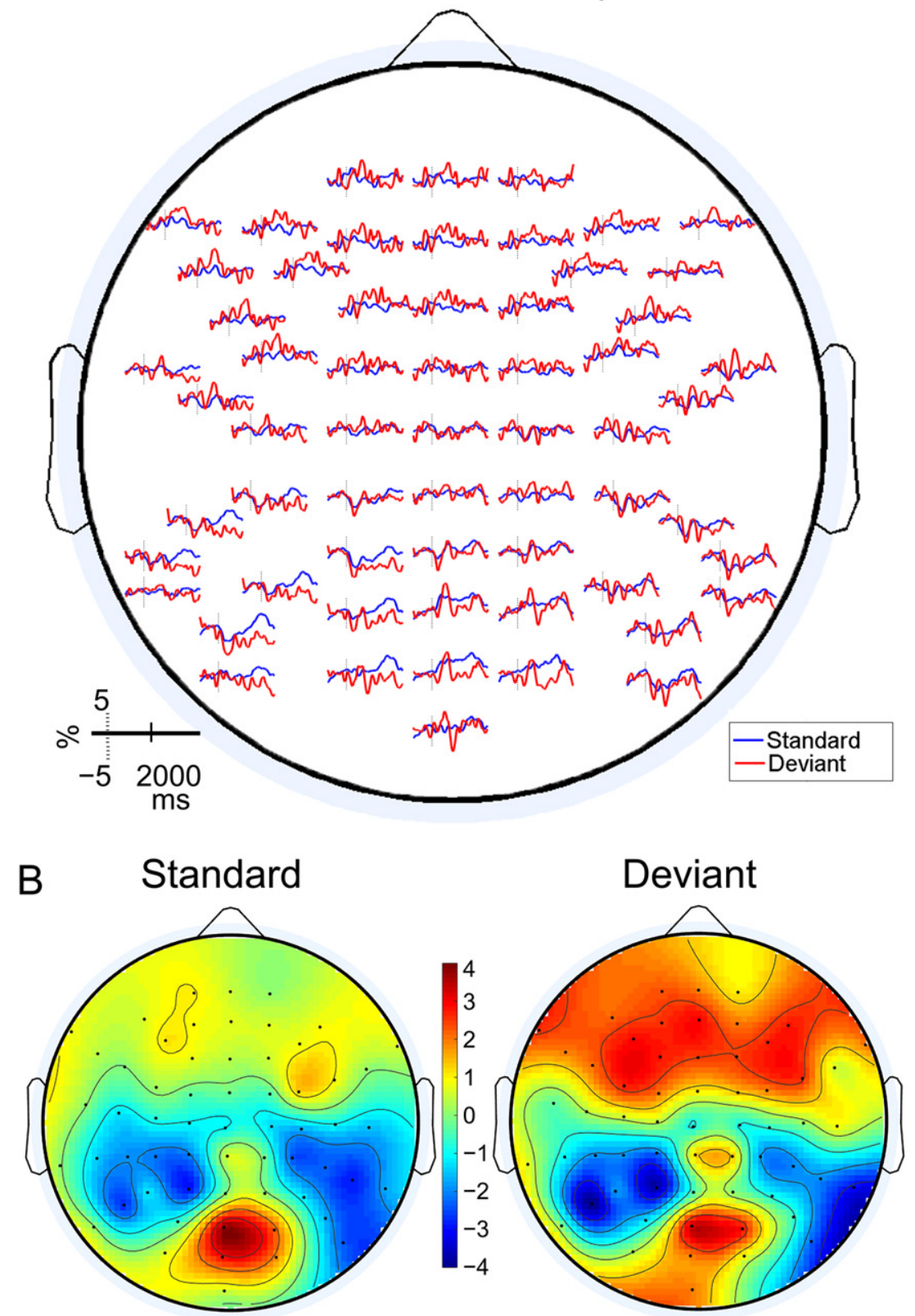

Fig. 7. ERD/ERS and topographies in sensor space for standard and deviant stimuli. A) Grand-average ERD/ERS responses across 20 subjects. B) Average of mean amplitudes in the time interval from 300 to $900 \mathrm{~ms}$ plotted as topographies across subjects.

standard and deviant stimuli. However, we found N1, P1 and P2 ERPrelated components typical for the visual stimuli (Omoto et al., 2010; Zalar et al., 2015), Fig. 8. Nevertheless, our approach revealed significant vMOR indicating that oscillations might be more susceptible to the detection of the deviant stimuli.

\section{Attention in $v M O R$}

In auditory MMN, a distraction task (such as reading a book, watching a movie) can be used to reduce attention of the subjects while the oddball stimuli are presented (MacLean and Ward, 2014; Näätänen et al., 2007). In visual MMN, distraction tasks are also used to reduce attention of the subject, for instance by utilizing the concurrent visual or motor task (Stefanics et al., 2014; Stothart and
Kazanina, 2013). Nevertheless, distraction tasks in visual studies could involve prediction and attention and therefore affect the EEG responses. In Stothart and Kazanina (2013), the oscillatory vMMN was studied using a target detection task in order to prevent the subjects from paying attention to the standard and deviant stimuli. In contrast to that study, where a motor reaction (to press a button) was required, here we used a passive paradigm where it was not necessary to perform/suppress any reaction. In our paradigm, the subjects were merely asked to look at the fixation cross.

In passive paradigms with a distraction task, in particular when the subjects are asked to react to rare target stimuli, the changedetection mechanism affects deviant stimuli more than standard ones; this is because deviant and target stimuli are more rare than the standards, see Müller and Keil (2004). For example, in the 

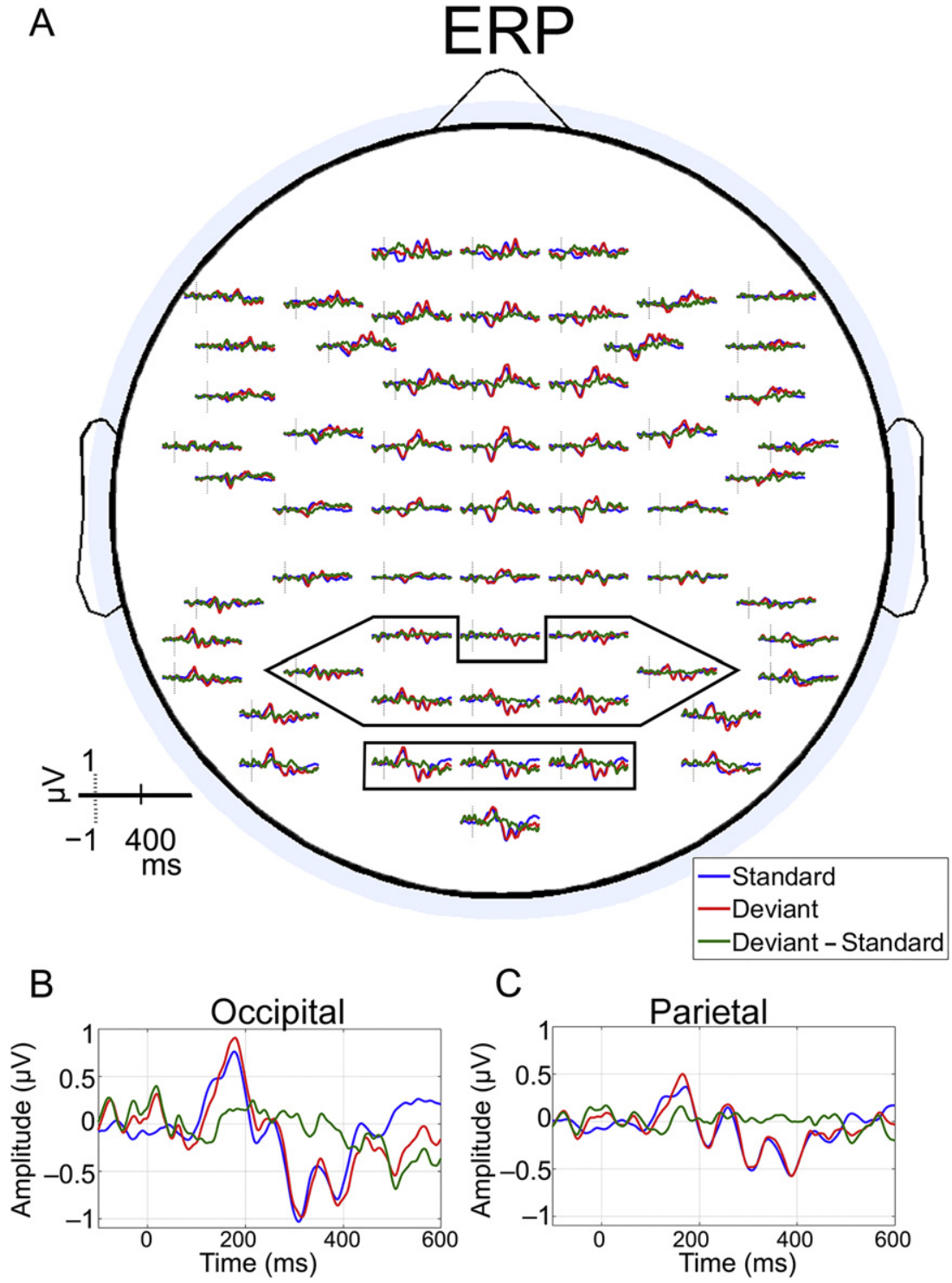

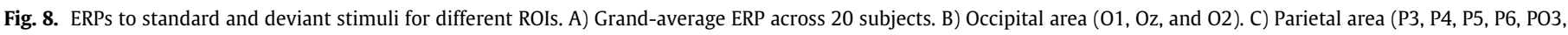
$\mathrm{POz}$, and $\mathrm{PO} 4)$.

paradigm of Stothart and Kazanina (2013) where the task was to press a button when the target stimuli were presented, the oscillatory responses to deviant stimuli could be due to the effect of attention.

We have not used any distraction task; the subjects were asked to focus on a fixation cross. Importantly, it has been shown that the peripheral visual stimulation effectively reduces the effect of attention in EEG responses (Stefanics et al., 2014). The EOG analysis did not reveal statistically significant differences between responses to standard and deviant stimuli. Given that the stimuli were presented at quite large angles $\left(59^{\circ}-90^{\circ}\right)$, a saccade in the direction of the deviant stimuli would have produced a strong EOG response, yet there were no differences detected in the amplitude of the EOG responses to the the two types of stimuli. The absence of differences between EOG responses shows that overt attention was not necessary to produce vMOR. Finally, in order to verify whether covert attention at least to some extent contributed to the generation of vMOR, we performed a behavioral experiment where we actually asked the subjects about the ratio of stimuli moving in different directions. If subjects had attended to the stimuli, they would have reported the ratios similar to the ratios found in the control experiment. Yet, in some cases the subjects were unable to indicate even approximately the correct ratio while having pronounced vMOR. In general, our results show that vMOR is unlikely to

Table 1

Behavioural responses in nine subjects with the instruction to attend or not to attend the stimuli. Ratio = standard:deviant.

\begin{tabular}{lll}
\hline \multicolumn{2}{l}{ Behavioural responses in nine subjects } \\
\hline Subjects & Responses without attention & Responses with attention \\
\hline S12 & $7: 1$ & $8: 1$ \\
S13 & $1: 1$ & $8: 1$ \\
S14 & $1: 5$ (opposite direction) & $7: 1$ \\
S15 & 1 (only standard stimuli) & $9: 1$ \\
S16 & $1: 1$ & $7: 1$ \\
S17 & $5: 1$ & $10: 1$ \\
S18 & $10: 1$ & $8: 1$ \\
S19 & $1: 4$ (opposite direction) & $9: 1$ \\
S20 & $2: 1$ & $8: 1$ \\
\hline
\end{tabular}




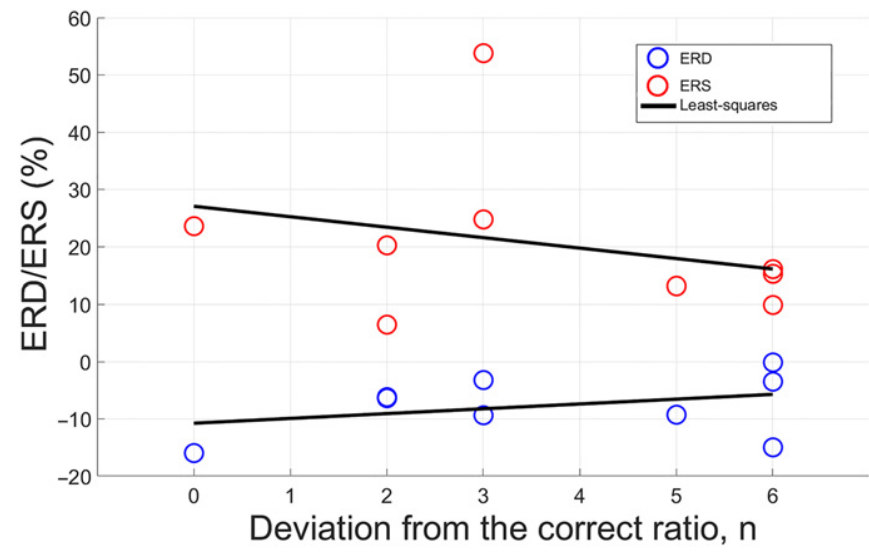

Fig. 9. Behavioural responses. The estimated ratio of stimuli as a function of ERD/ERS peak amplitudes for nine subjects. Deviation from the correct ratio is expressed as: $7 \pm \mathrm{n}: 1$.

be strongly influenced by attention. On the other hand, ERP amplitudes are known to be modulated by attention. The N100 component is sensitive to attention and is generated in the auditory cortex (Hillyard et al., 1973; Meyberg et al., 2015; Näätänen and Picton, 1987). Visual spatial attention enhances N100 and P100 amplitudes in the visual cortex (Herrmann and Knight, 2001; Hillyard and Anllo-Vento, 1998; Koivisto and Revonsuo, 2010). Another prominent example of ERP with strong attentional modulation is P300, which is usually observed in the odd-ball paradigms where attention is paid to the target stimuli (Harmony et al., 2000; Polich, 2007). Interestingly, we did not observe any significant differences in the ERP to standard and deviant stimuli in any of the analyzed ROIs. Given that ERP amplitudes are reliable indicators of attention, this result is consistent with the idea that attention was unlikely to be involved in the generation of vMOR. Overall, one cannot completely exclude the effect of attention, but this is rather a common criticism relating to many MMN studies.

Our results indicate that the vMOR is likely to be due to central processing leading to the detection of the visual deviant stimuli. To our knowledge, this is the first study where vMOR has been demonstrated using a passive paradigm without a distraction task.

\section{ERD/ERS in alpha oscillations}

Alpha oscillations are thought to reflect dynamic engagement of specific cortical areas into a given task (Jensen et al., 2014; Jensen and Mazaheri, 2010; Klimesch, 2012; Palva and Palva, 2007). In this framework, ERD reflects active neuronal processing (Pfurtscheller and Lopes da Silva, 1999), while ERS rather relates to the inhibition of task-irrelevant information processing (Jensen et al., 2014; Jensen and Mazaheri, 2010; Palva and Palva, 2007). The CSP method was capable of recovering ERD and ERS even if the corresponding topographies of the sources heavily overlap. In fact, in a computational model it has been even shown that ERD and ERS might simultaneously coexist in the form of "focal ERD/surround ERS" (Suffczynski et al., 2001). Such juxtaposition of ERD and ERS thus most likely reflects prioritizing of task-relevant information processing with a simultaneous suppression of task-irrelevant activity (Worden et al., 2000). In the present study, ERD/ERS started early and only the peak latencies were in the time range of 500-730 ms. This in turn indicates that the amplitude of alpha oscillations can change quickly to the deviant stimuli and that only the full development of the response requires additional few hundreds of milliseconds.

It has been shown that the dynamics of ERPs and oscillations are different even when both can originate from the same sources (Hanslmayr et al., 2007). While the duration of ERPs is not longer than 600-800 ms, visual stimulation and working memory can induce changes in the alpha band oscillations lasting for $3 \mathrm{~s}$ (Chen et al., 2015). The differences in the duration of ERPs and ERD/ERS have been demonstrated in many studies with different kind of stimuli and tasks; in general ERD/ERS usually peak later than the ERP components (Anderson and Ding, 2011; Deiber et al., 2010; Toledo et al., 2016). In our study, ERD/ERS latencies were longer compared to the previously reported vMMN latencies; however, our results are in line with previous findings where oscillatory responses were late compared to ERPs. In addition, ERD/ERS in our study started already at $300 \mathrm{~ms}$, which suggests an earlier onset of the neuronal processes related to vMOR.

An empirical study, combined with computational modeling, showed that the generation of the responses to deviant stimuli in auditory MMN paradigm was mediated by recurrent and reentrant neuronal dynamics (Garrido et al., 2007), thus emphasizing the role of the feedback connections. Moreover, a recent study on monkeys (van Kerkoerle et al., 2014), showed that while feed-forward connections in the visual system are related to gamma oscillations, feedback connections are primarily based on alpha oscillations. If one assumes that not only the generation of auditory but also visual mismatch responses require feedback connections, then the amplitude dynamics of alpha oscillations might indeed mediate the detection of deviant stimuli, which would be in agreement with the result of the present study showing a clear presence of stronger oscillatory responses to deviant stimuli.

One of the limitations of our study is the absence of differences in evoked responses to standard and deviant stimuli. We believe that it might be due to the small size of ERP to peripheral stimuli used in the present study. This might also be due to a relatively small number of epochs, which, however, was sufficient for the detection of changes in ERD/ERS.

\section{Conclusion}

To summarize, we showed that, compared to standard visual stimuli, the deviant stimuli produced stronger increase and decrease of occipito-parietal alpha oscillations in a passive paradigm. Such oscillatory responses indicate that the stimulus change can be reflected not only in evoked activity, as shown in previous studies, but also in the amplitude of ongoing neuronal oscillations.

Supplementary data to this article can be found online at http:// dx.doi.org/10.1016/j.neuroimage.2016.07.024.

\section{Acknowledgments}

The authors thank the Academy of Finland for funding. S.T. thanks Center for International Mobility (CIMO), Finland for funding. V.V.N. acknowledges support from the Russian Academic Excellence Project '5-100'.

\section{References}

Amano, K., Nishida, S., Takeda, T., 2006. MEG responses correlated with the visual perception of velocity change. Vis. Res. 46 (3), 336-345.

Amenedo, E. Pazo-Alvarez, P. Cadaveira, F. 2007. Vertical asymmetries in pre-attentive detection of changes in motion direction. Int. J. Psychophysiol. 64 (2), 184-189.

Anderson, K., Ding, M., 2011. Attentional modulation of the somatosensory mu rhythm. Neuroscience 180, 165-180.

Babiloni, C., Miniussi, C., Babiloni, F., Carducci, F., Cincotti, F., Del Percio, C., Sirello, G., Fracassi, C., Nobre, A.C., Rossini, P.M., 2004. Sub-second temporal attention modulates alpha rhythms. A high-resolution EEG study. Cogn. Brain Res. 19 (3), 259-268.

Blankertz, B., Tomioka, R., Lemm, S., Kawanabe, M., Müller, K.-R., 2008. Optimizing spatial filters for robust EEG single-trial analysis. IEEE Signal Process. Mag. 25 (1) 41-56. 
Bočková, M., Chládek, J., Šimová, L., Jurák, P., Halámek, J., Rektor, I., 2013. Oscillatory changes in cognitive networks activated during a three-stimulus visual paradigm: an intracerebral study. Clin. Neurophysiol. 124 (2), 283-291.

Chen, Y., Chen, X., Kuang, C., Huang, X., 2015. Neural oscillatory correlates of duration maintenance in working memory. Neuroscience 290, 389-397.

Czigler, I., 2014. Visual mismatch negativity and categorization. Brain Topogr. 27 (4) 590-598.

Deiber, M.-P., Rodriguez, C., Jaques, D., Missonnier, P., Emch, J., Millet, P., Gold, G., Giannakopoulos, P., Ibanez, V., 2010. Aging effects on selective attention-related electroencephalographic patterns during face encoding. Neuroscience 171 (1), 173-186.

Fuentemilla, L., Marco-Pallarés, J., Münte, T., Grau, C., 2008. Theta EEG oscillatory activity and auditory change detection. Brain Res. 1220, 93-101.

Garrido, M.I., Kilner, J.M., Kiebel, S.J., Friston, K.J., 2007. Evoked brain responses are generated by feedback loops. Proc. Natl. Acad. Sci. 104 (52), 20961-20966.

Hanslmayr, S., Klimesch, W., Sauseng, P., Gruber, W., Doppelmayr, M., Freunberger, R., Pecherstorfer, T., Birbaumer, N., 2007. Alpha phase reset contributes to the generation of ERPs. Cereb. Cortex 17 (1), 1-8.

Harmony, T., Bernal, J., Fernández, T., Silva-Pereyra, J., Fernandez-Bouzas, A., Marosi, E., Rodríguez, M., Reyes, A., 2000. Primary task demands modulate P3a amplitude. Cogn. Brain Res. 9 (1), 53-60.

Haufe, S., Dähne, S., Nikulin, V.V., 2014. Dimensionality reduction for the analysis of brain oscillations. Neuroimage 101, 583-597.

Herrmann, C.S., Knight, R.T., 2001. Mechanisms of human attention: event-related potentials and oscillations. Neurosci. Biobehav. Rev. 25 (6), 465-476.

Hillyard, S.A., Anllo-Vento, L., 1998. Event-related brain potentials in the study of visual selective attention. Proc. Natl. Acad. Sci. 95 (3), 781-787.

Hillyard, S.A., Hink, R.F., Schwent, V.L., Picton, T.W., 1973. Electrical signs of selective attention in the human brain. Science 182 (4108), 177-180.

Hsiao, F.-J., Wu, Z.-A., Ho, L.-T., Lin, Y.-Y., 2009. Theta oscillation during auditory change detection: an MEG study. Biol. Psychol. 81 (1), 58-66.

Jensen, O., Gips, B., Bergmann, T.O., Bonnefond, M., 2014. Temporal coding organized by coupled alpha and gamma oscillations prioritize visual processing. Trends Neurosci. 37 (7), 357-369.

Jensen, O., Mazaheri, A., 2010. Shaping functional architecture by oscillatory alpha activity: gating by inhibition. Front. Hum. Neurosci. 4 (4), 1-8.

Kimura, M., 2012. Visual mismatch negativity and unintentional temporal-context-based prediction in vision. Int. J. Psychophysiol. 83 (2), 144-155.

Klimesch, W.,2012. Alpha-band oscillations, attention, and controlled access to stored information. Trends Cogn. Sci. 16 (12), 606-617.

Ko, D., Kwon, S., Lee, G.-T., Im, C.H., Kim, K.H., Jung, K.-Y., 2012. Theta oscillation related to the auditory discrimination process in mismatch negativity: oddball versus control paradigm. J. Clin. Neurol. 8 (1), 35-42.

Koivisto, M., Revonsuo, A., 2010. Event-related brain potential correlates of visual awareness. Neurosci. Biobehav. Rev. 34 (6), 922-934.

Kuldkepp, N., Kreegipuu, K., Raidvee, A., Näätänen, R., Allik, J., 2013. Unattended and attended visual change detection of motion as indexed by event-related potentials and its behavioral correlates. Front. Hum. Neurosci. 7, 1-9.

Lamberty, K. Gobbelé, R., Schoth, F., Buchner, H., Waberski, T.D., 2008. The tempora pattern of motion in depth perception derived from ERPs in humans. Neurosci. Lett. 439 (2), 198-202.

MacLean, S.E., Ward, L.M., 2014. Temporo-frontal phase synchronization supports hierarchical network for mismatch negativity. Clin. Neurophysiol. 125 (8), 1604-1617.

Meyberg, S., Werkle-Bergner, M., Sommer, W., Dimigen, O., 2015. Microsaccaderelated brain potentials signal the focus of visuospatial attention. Neuroimage 104, 79-88.

Müller, M.M., Keil, A., 2004. Neuronal synchronization and selective color processing in the human brain. J. Cogn. Neurosci. 16 (3), 503-522.
Näätänen, R., Kujala, T., Winkler, I., 2011. Auditory processing that leads to conscious perception: a unique window to central auditory processing opened by the mismatch negativity and related responses. Psychophysiology 48 (1), 4-22.

Näätänen, R., Paavilainen, P., Rinne, T., Alho, K., 2007. The mismatch negativity (MMN) in basic research of central auditory processing: a review. Clin. Neurophysiol. 118 (12), 2544-2590.

Näätänen, R., Picton, T., 1987. The N1 wave of the human electric and magnetic response to sound: a review and an analysis of the component structure. Psychophysiology 24 (4), 375-425

Omoto, S., Kuroiwa, Y., Otsuka, S., Baba, Y., Wang, C., Li, M., Mizuki, N., Ueda, N., Koyano, S., Suzuki, Y., 2010. P1 and P2 components of human visual evoked potentials are modulated by depth perception of 3-dimensional images. Clin. Neurophysiol. 121 (3), 386-391.

Palva, S., Palva, J.M., 2007. New vistas for $\alpha$-frequency band oscillations. Trends Neurosci. 30 (4), 150-158.

Pazo-Alvarez, P., Amenedo, E., Cadaveira, F., 2004. Automatic detection of motion direction changes in the human brain. Eur. J. Neurosci. 19 (7), 1978-1986.

Pazo-Alvarez, P., Cadaveira, F., Amenedo, E., 2003. MMN in the visual modality: a review. Biol. Psychol. 63 (3), 199-236.

Pfurtscheller, G., Lopes da Silva, F.H., 1999. Event-related EEG/MEG synchronization and desynchronization: basic principles. Clin. Neurophysiol. 110 (11), 1842-1857.

Polich, J., 2007. Updating P300: an integrative theory of P3a and P3b. Clin. Neurophysiol. 118 (10), 2128-2148.

Roach, B.J., Mathalon, D.H., 2008. Event-related EEG time-frequency analysis: an overview of measures and an analysis of early gamma band phase locking in schizophrenia. Schizophr. Bull. 34 (5), 907-926.

Sauseng, P., Klimesch, W., Stadler, W., Schabus, M., Doppelmayr, M., Hanslmayr, S., Gruber, W., Birbaumer, N., 2005. A shift of visual spatial attention is selectively associated with human EEG alpha activity. Eur. J. Neurosci. 22 (11), 2917-2926.

Sokolov, E.N., Spinks, J.A., Näätänen, R., Lyytinen, H., 2002. The Orienting Response in Information Processing. Lawrence Erlbaum Associates Publishers.

Stefanics, G., Csukly, G., Komlósi, S., Czobor, P., Czigler, I., 2012. Processing of unattended facial emotions: a visual mismatch negativity study. Neuroimage 59 (3), 3042-3049.

Stefanics, G., Kremláček, J., Czigler, I., 2014. Visual mismatch negativity: a predictive coding view. Front. Hum. Neurosci. 8, 1-19.

Stothart, G., Kazanina, N., 2013. Oscillatory characteristics of the visual mismatch negativity: what evoked potentials aren't telling us. Front. Hum. Neurosci. 7 (426), $1-9$

Suffczynski, P., Kalitzin, S., Pfurtscheller, G., Da Silva, F.L., 2001. Computational model of thalamo-cortical networks: dynamical control of alpha rhythms in relation to focal attention. Int. J. Psychophysiol. 43 (1), 25-40.

Thut, G., Miniussi, C., Gross, J., 2012. The functional importance of rhythmic activity in the brain. Curr. Biol. 22 (16), R658-R663.

Toledo, D.R., Manzano, G.M., Barela, J.A., Kohn, A.F., 2016. Cortical correlates of response time slowing in older adults: ERP and ERD/ERS analyses during passive ankle movement. Clin. Neurophysiol. 127 (1), 655-663.

van Kerkoerle, T., Self, M.W., Dagnino, B., Gariel-Mathis, M.-A., Poort, J., van der Togt, C., Roelfsema, P.R., 2014. Alpha and gamma oscillations characterize feedback and feedforward processing in monkey visual cortex. Proc. Natl. Acad. Sci. 111 (40), $14332-14341$.

Worden, M.S., Foxe, J.J., Wang, N., Simpson, G.V., 2000. Anticipatory biasing of visuospatial attention indexed by retinotopically specific $\alpha$-band electroencephalography increases over occipital cortex. J. Neurosci. 20 (RC63), 1-6.

Zalar, B., Martin, T., Kavcic, V., 2015. Cortical configuration by stimulus onset visual evoked potentials (SO-VEPs) predicts performance on a motion direction discrimination task. Int. J. Psychophysiol. 96 (3), 125-133. 